\title{
An imaging technique for subsurface faults using Teleseismic-Wave Records II -Improvement in the detectability of subsurface faults-
}

\author{
Takumi Murakoshi ${ }^{1}$, Hiroshi Takenaka ${ }^{1}$, and Daisuke Suetsugu ${ }^{2}$ \\ ${ }^{1}$ Department of Earth and Planetary Sciences, Kyushu University, Fukuoka 812-8581, Japan \\ ${ }^{2}$ IISEE, Building Research Institute, Tachiharal, Tsukuba 305-0032, Japan \\ (Received April 15, 1999; Revised October 13, 1999; Accepted October 13, 1999)
}

\begin{abstract}
We have developed an imaging algorithm for subsurface faults on the basis of the steepest descent method in our previous paper. In this paper we introduce two new techniques, the multi-event stacking and multi-master stacking, to improve detectability for subsurface faults. The multi-event stacking is a technique using data from plural earthquakes with different incident angles. The multi-master stacking is a technique using data of a single earthquake, in which the images obtained by employing different master receiver are stacked. We examined the feasibility of our approach using several trial models and synthetic data with different signal to noise ratios, different pulse durations and different incident angles in the two dimensional $S H$ cases. In the numerical experiments, we could recover the image of the subsurface fault for all the trial models used, which indicates our method has a potential for sensing real subsurface faults.
\end{abstract}

\section{Introduction}

Detection of subsurface faults is an essential problem in the viewpoint of earthquake disaster prevention, because there may exist many active subsurface faults that are not detected by geological investigations or studies of air photographs. Takenaka et al. (1996) proposed a new method for imaging subsurface faults using teleseismicwave records. They did not determine an entire velocity structure, but location and size of a subsurface fault. The imaging algorithm is based on the steepest descent method. This is similar to the waveform inversion of reflection data (e.g., Tarantola, 1984), but no iteration is required in Takenaka et al. (1996); only one forward and reverse-time computation of the wavefield are made. Takenaka et al. (1996) demonstrated the feasibility of the method using synthetic noise-free data of a vertically incident plane $S H$ wave from one event. Murakoshi et al. (1996) performed some numerical experiments on imaging a subsurface fault, using several trial models and a vertically incident synthetic $S H$ plane wave without noise, and examined the effect of the trial models on the detectability of subsurface faults. They also proposed a practical technique to estimate the incident wavefield from the observed data.

For real data processing, we need a linear array of suitably spaced receivers for the size of the target structure. The minimum size of the detectable fault increases inverse proportionally to the receiver spacing. The scale of the target which can be to image also depends on the size of earthquakes available for the imaging and the frequency band of the seismometer and the recording system. Since we have to avoid waveforms complicated by complex

Copy right $\odot$ The Society of Geomagnetism and Earth, Planetary and Space Sciences (SGEPSS); The Seismological Society of Japan; The Volcanological Society of Japan; The Geodetic Society of Japan; The Japanese Society for Planetary Sciences. source process to focus on the effects of subsurface structure, our imaging technique may be applicable to earthquakes smaller than M6, which usually have rather simple source time functions.

In the present study we introduce two new techniques for the method: the multi-event stacking and multi-master stacking, to obtain clearer image of subsurface faults than the single-event method investigated by Takenaka et al. (1996) and Murakoshi et al. (1996). In the multi-event stacking, images obtained from plural events are stacked to improve the signal-to-noise ratio in the final image. In the multi-master stacking, we use data from a single event, but stack multiple images from multiple master receivers to obtain a final image. The master receivers are employed for removing the initial phase from the observed records, estimation of the incident wavefield and synchronizing the observed and theoretical waveform data. We perform numerical experiments using the synthetic $S H$ data with different signal-to-noise ratios, different pulse durations and different incident angles, and compare the results of the proposed techniques with that of the single-event method. Results of the experiments show that the stacking techniques improve the detectability of underground faults over the single-event method.

\section{Imaging Method}

Figure 1 shows the procedure of the imaging method in this study. The shaded boxes in Fig. 1 show the procedure of the imaging method in Takenaka et al. (1996) and Murakoshi et al. (1996). They use a trial model and a master receiver to calculate the steepest descent direction for the rigidity distribution from waveform records of an earthquake for identifying faults. Expanding this approach we introduce two new techniques to improve the detectability of subsurface faults: the multi-event stacking 


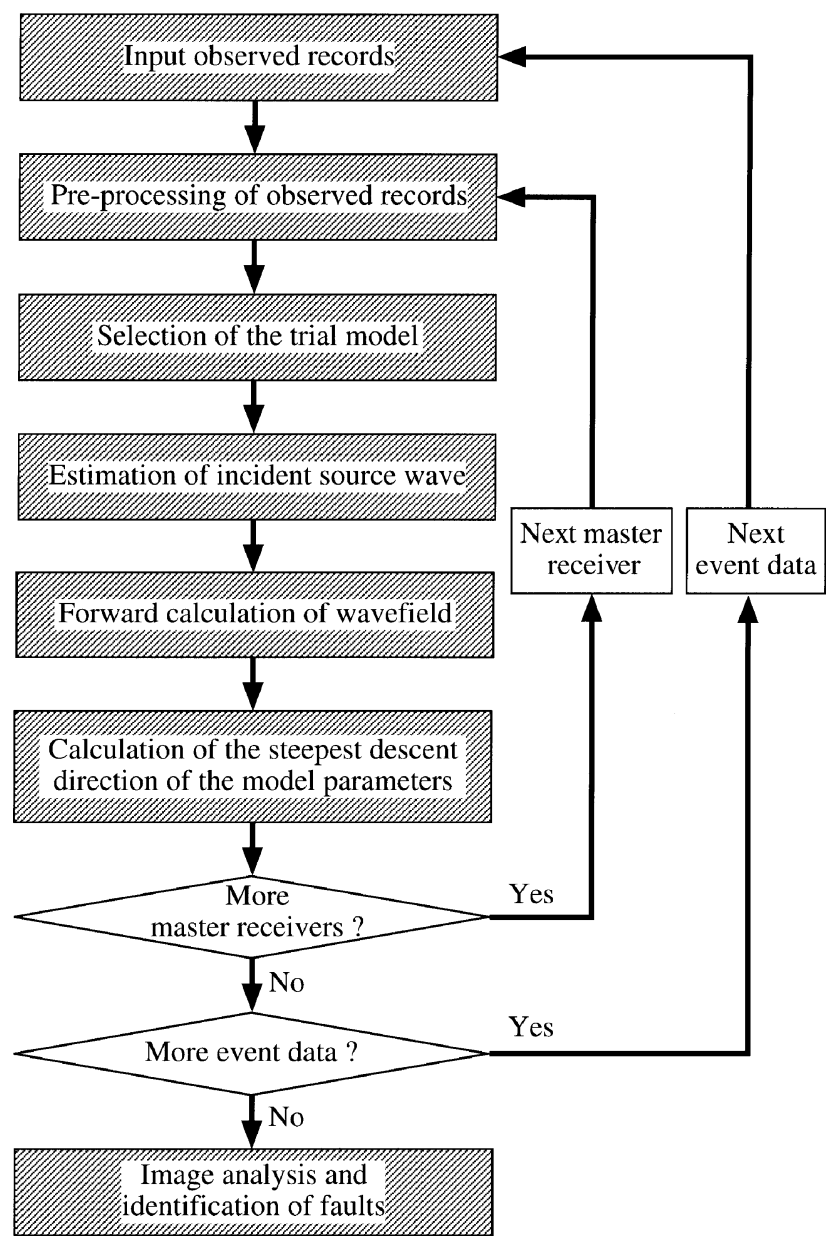

Fig. 1. The procedure of the imaging method in this study. The shaded boxes represent our previous imaging method, and the processes shown in the white boxes are added to them in this study.

and the multi-master stacking. The multi-event stacking adds up the images derived from different earthquakes with different incident angles. The multi-master stacking is a technique using data of a single earthquake, in which the images obtained by using different master receivers are stacked. In practice the multi-event stacking repeats the process of "Next event data" in Fig. 1 for all the events to obtain an image from each event data, and stacks all the derived images. The multi-master stacking repeats the process of "Next master receiver" in Fig. 1 to obtain an image using each "master receiver," and then stacks all the images.

The pre-processing removes the initial phase from these observed records. The later phases in the observed data have much more information on the layering structure and its offset due to the fault than the initial phase has. If the pre-processing is not performed, the information of the later phases is masked by the much larger amplitude of the initial phase compared to those of the later phases, so that it is difficult to detect the target structure. Takenaka et al. (1996) discussed the effects of the pre-processing as well as the method. We assume the trial model of the subsurface structure and then estimate an incident source wave from the initial phase of the record of a site selected as "master

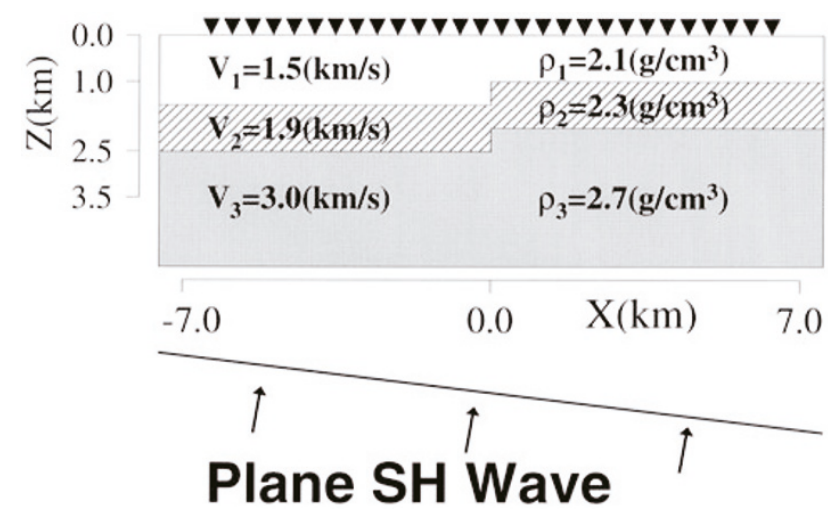

Fig. 2. Subsurface fault model used as the "true" structure, which has a vertical fault with an offset of $500 \mathrm{~m}$. This model assumed an incident wave to be the upgoing plane $S H$ wave. The receivers are denoted by the solid triangles, located with an interval of $500 \mathrm{~m}$. The whole size of the computational domain is $20 \mathrm{~km}$ (horizontally) $\times 5$ $\mathrm{km}$ (vertically).

receiver". The seismic response of the trial model is calculated by the finite-difference method in forward calculation of wavefield. We calculate the steepest descent direction of the model parameters from residuals between observed and calculated waveforms to identify faults.

We can retrieve the incident waveform in the following way: First, analyze the observed records using the semblance technique to estimate the ray parameter of the incident wave. Second transfer the initial phase at surface downward by deconvolution using the response of the laterally homogeneous trial model for the estimated ray parameter to obtain the incident wave at the bottom of the model. Details are described in Murakoshi et al. (1996).

\section{Numerical Experiments}

\subsection{General imaging procedure for subsurface fault}

We employ the model in Fig. 2 as the true structure for creating synthetic test data, which is the same as used by Murakoshi et al. (1996). This model includes two horizontal layers and a vertical fault with a vertical offset of $500 \mathrm{~m}$ and total size of $1.5 \mathrm{~km}$. There are 28 receivers deployed at intervals of $500 \mathrm{~m}$ from $x=-7 \mathrm{~km}$ to $x=7 \mathrm{~km}$ at the free surface. The incident source wave is a plane $\mathrm{SH}$ wave whose time function is bell-shaped with a pulse duration $\tau=0.5$ or $0.8 \mathrm{~s}$. The incident angle is $\pm 5^{\circ}, \pm 10^{\circ}$, $\pm 15^{\circ}, \pm 20^{\circ}, \pm 30^{\circ}$.

To examine feasibility of our imaging procedure, we produce two kinds of synthetic data by the finite-difference method (Boore, 1972), which are used as observed records: noise-free data and data containing noise. Figure 3 shows examples of synthetic data (the incident angle $+15^{\circ}, \tau=$ $0.5 \mathrm{~s})$. The reverberations after the first $S H$ wave bear information on the layering of the structure. An offset in time of the reverberations at $x=0$ indicates an offset in the layering there. We have designed the noise with the same power spectral shape as the incident signal and with random phase, so that the signal reflected from the layered structure may be difficult to be identified in each record with large noise (see Fig. 3(b)). We produce different 

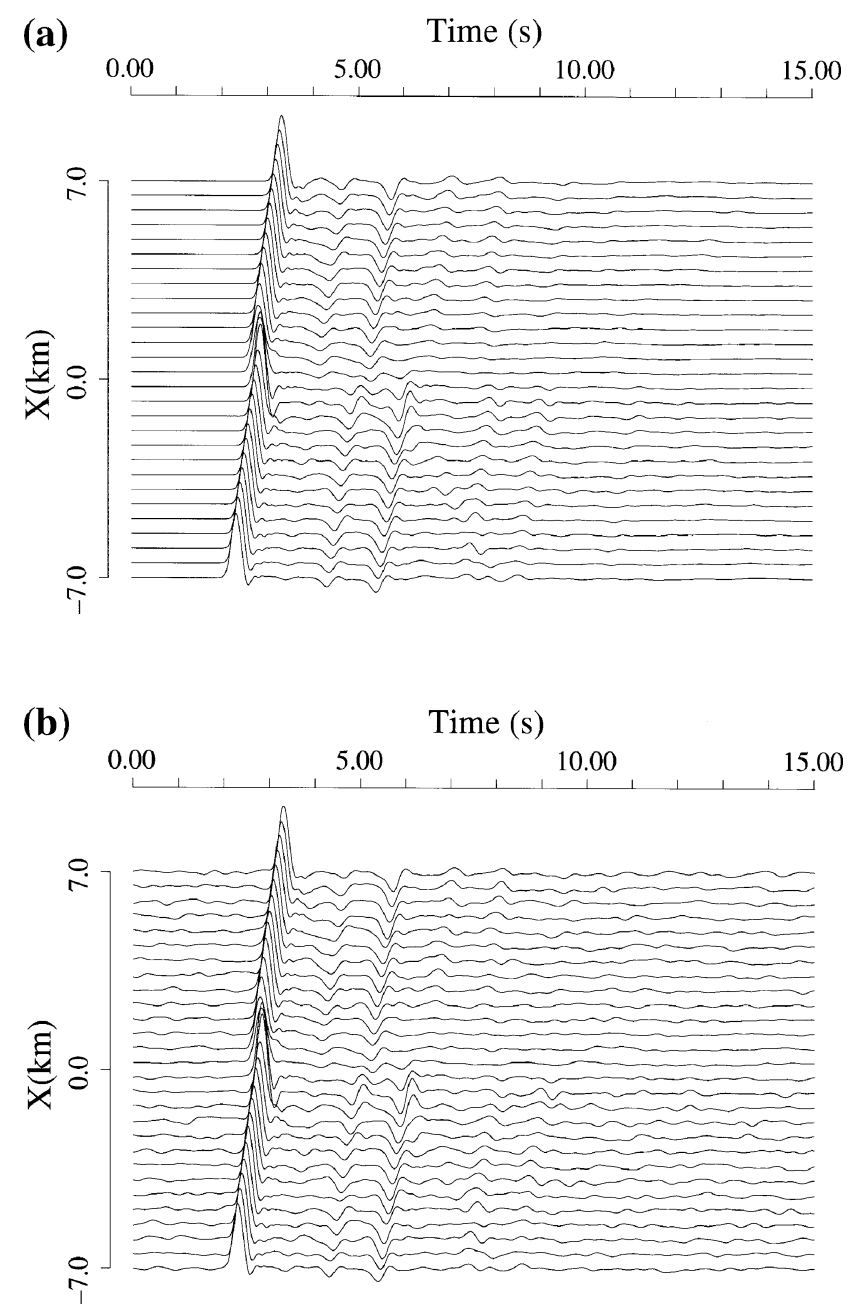
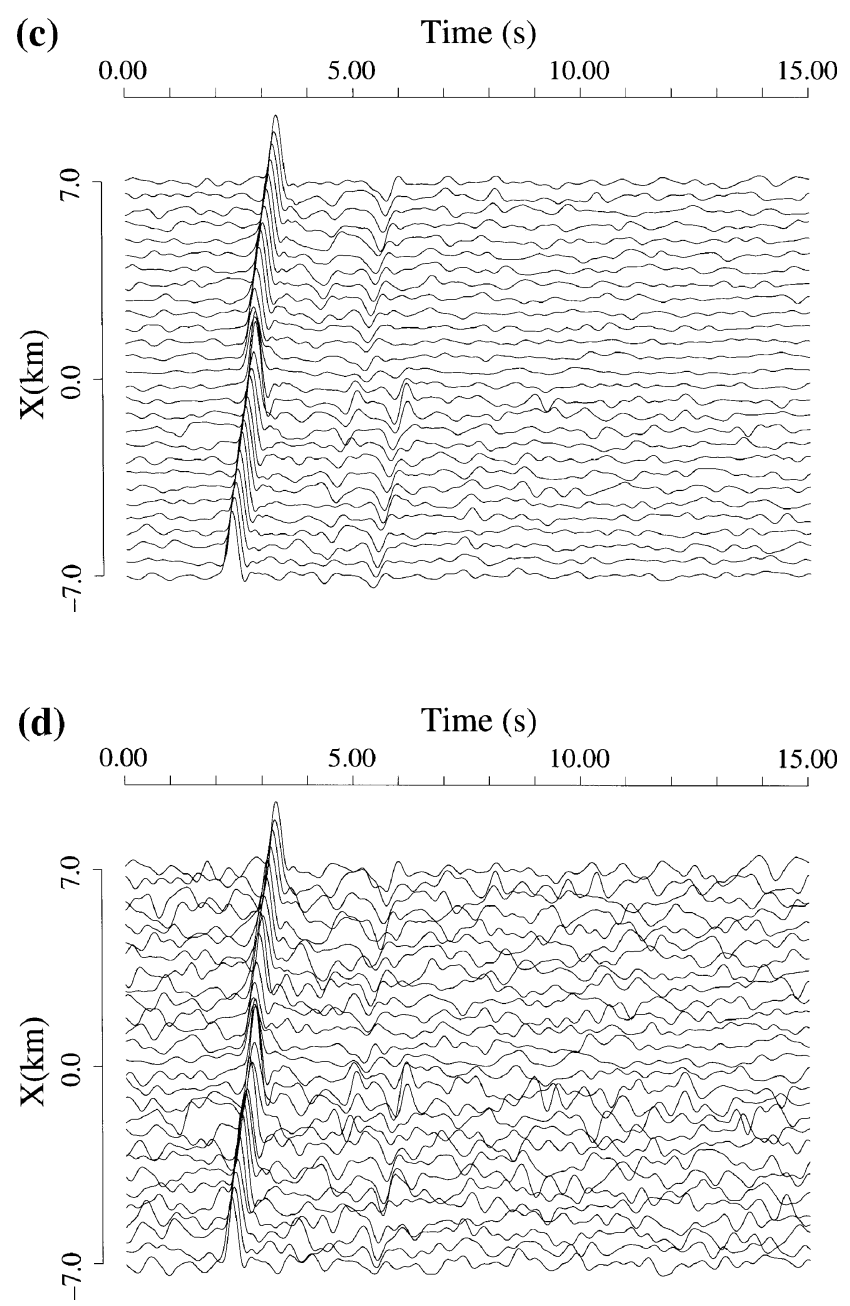

Fig. 3. Examples of the "observed" records for the incident angle of $+15^{\circ}$. A bell-shaped time function with a pulse duration $(\tau)$ of $0.5 \mathrm{~s}$ and unit area is used. (a) The noise-free data. (b), (c) and (d) are data containing noise, which are generated by summing signals (Fig. 3(a)) and random noise at three different N/S ratios. The N/S ratios are (b) 0.1 , (c) 0.2 and (d) 0.5 . The N/S ratio is defined to be the maximum amplitude of the signal divided by 1.4 times the rms amplitude of the noise.

noise for different receivers, and then add each noise to noise-free observed records. Details are shown in the Appendix. Signal-to-noise ratio of the data is defined by the relation $\mathrm{N} / \mathrm{S}=\left(1.4 \times\right.$ noise $\left._{\mathrm{rms}}\right) /\left(\right.$ data $\left._{\max }\right)($ Kong et al. , 1985), where data $_{\max }$ and noise $_{\text {rms }}$ are the maximum amplitude of pre-processed record and the root mean square of the random noise, respectively. We assume N/ $\mathrm{S}=0.0,0.1,0.2$ or 0.5 . For pre-processing of noisy observed records, we apply a cosine-taper to the data before the initial phase to remove the trivial noise.

We first calculate seismic wavefield forward in time using a trial model (refer to Fig. 1). The incident wave is estimated from an observed record as described in Murakoshi et al. (1996). We employ the five trial models shown in Fig. 4 to study effects of trial model on the final image, because the trial model constructed by available geological and geophysical data has uncertainty in real cases.

We next calculate residuals for a trial model. The rigidity adjustment distribution $\tilde{\delta \mu}(\boldsymbol{x})$ (the steepest descent direction) to a trial model is obtained from the residuals by the following representation (Takenaka et al., 1996):

$$
\tilde{\delta \mu}(\boldsymbol{x})=-\sum_{e=1}^{N_{e}} \int_{0}^{T} d t \nabla v_{t h}(\boldsymbol{x}, t)^{(e)} \cdot \nabla \psi(\boldsymbol{x}, t)^{(e)},
$$

where $N_{e}$ is the number of events (earthquakes), $T$ is the time window used for imaging, $\boldsymbol{x}$ is the spatial position vector, $v_{\text {th }}$ is the $S H$ wavefield obtained by performing a forward calculation for a trial model. $\psi$ is the residual wavefield which is obtained by solving the following equation in reverse-time order from $t=T$ :

$$
\rho \frac{\partial^{2}}{\partial t^{2}} \psi(\boldsymbol{x}, t)-\nabla \cdot\left[\mu(\boldsymbol{x})_{0} \nabla \psi(\boldsymbol{x}, t)\right]=\sum_{r=1}^{N_{r}} \delta\left(\boldsymbol{x}-\boldsymbol{x}_{r}\right) \delta v\left(\boldsymbol{x}_{r}, t\right),
$$

where $\mu(\boldsymbol{x})_{0}$ is the rigidity distribution of the trial model, $\rho$ is the density, $N_{r}$ is the number of receivers, $\boldsymbol{x}_{r}$ are the spatial position vectors of receivers and $\delta v\left(\boldsymbol{x}_{r}, t\right)$ are the residual data at the receivers, i.e., difference between the observed and the calculated pre-processed waveform. If 


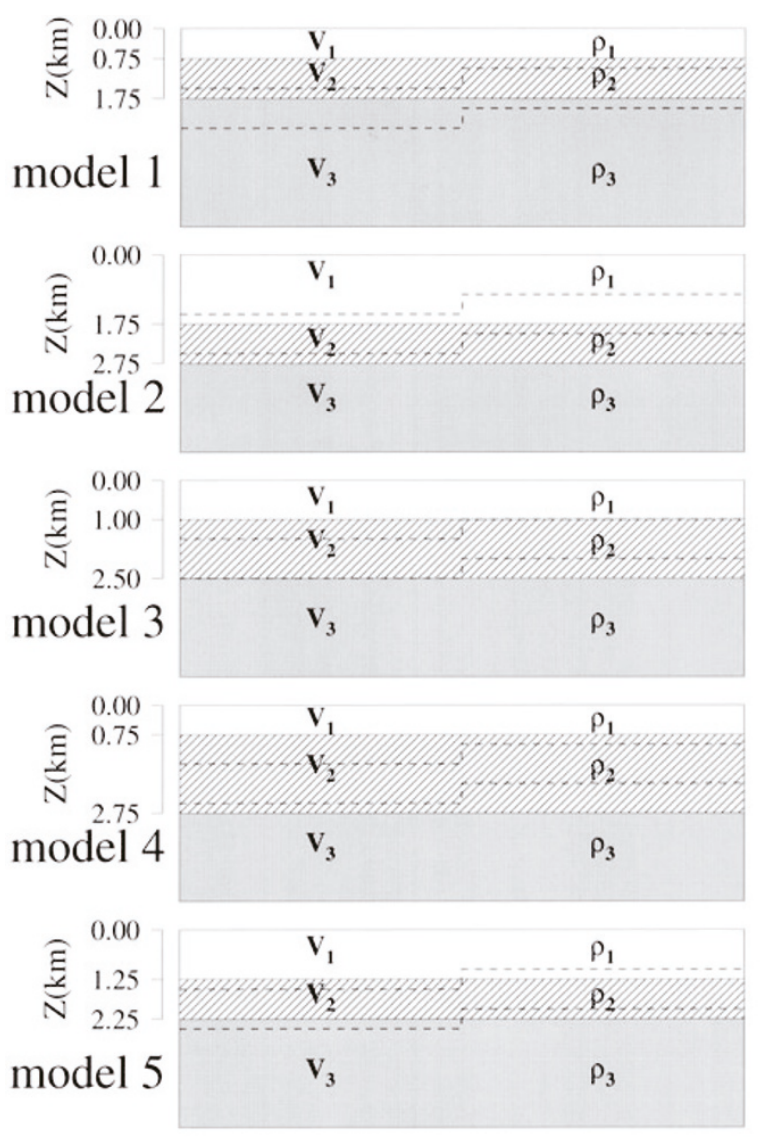

(a)

Fig. 4. Five different trial models used in the numerical experiments. They are horizontally layered structures without fault. The dashed line is the true structure shown in Fig. 2. Trial models 1 to 5 are shown in (a) to (e), respectively.

we would like to know the velocity structure, we had to estimate a proper scaling factor (step length in the direction of the steepest descent) to update the rigidity distribution and perform the iteration. The target of our approach is, however, to detect and locate the fault, not to reconstruct the underground velocity structure. Our approach therefore uses the steepest descent direction (see Takenaka et al., 1996 for detail).

\subsection{Multi-event stacking}

In the multi-event stacking, we stack the images calculated by Eq. (1) for different events (i.e., $N_{e} \geq 2$ ). We illustrate the multi-event stacking in Figs. 5 and 6(c). Figure 5 shows each image obtained from the single-event noise-free data (i.e., $N_{e}=1$ ) for each of the events with incident angle of $\pm 5^{\circ}, \pm 10^{\circ}, \pm 15^{\circ}, \pm 20^{\circ}$ or $\pm 30^{\circ}$ and $\tau=0.5$ $\mathrm{s}$ using trial model 3 . Figure 6(c) is the image obtained by the multi-event stacking, in which all the images in Fig. 5 are stacked.

The results of the imaging procedure characteristically emphasize the image around receivers (e.g., Gauthier $e t$ al., 1986), because the receivers are point sources (i.e., singular points) in the residual wavefield computation (Eq. (2)). It is then difficult to obtain the correct near surface images due to contamination of large artifacts caused by the near surface singular points. Also, the target of our imaging is not that shallow. Therefore, only the image in the area $-7 \leq x \leq 7(\mathrm{~km}), 0.6 \leq z \leq 3.5(\mathrm{~km})$ is shown (The area $0 \leq z \leq 0.6(\mathrm{~km})$ is also painted with green for easy comparison with Fig. 2). The results in our approach do not give the values of rigidity adjustment to the trial model, but give the direction for the adjustment (steepest descent direction) that has enough information to detect the fault in our approach. We have normalized the values of the steepest descent direction by its maximum value to display them up to the scale of two thirds of the maximum absolute value. So the colorscales in Figs. 5 and 6 indicate the relative strength of positive and negative adjustment.

We also show the corresponding images derived by a single event imaging (incident angle of $+15^{\circ}, \tau=0.5 \mathrm{~s}, \mathrm{~N} /$ $S=0.0)$ in Fig. 7 to see the effect of the multi-event stacking. The interfaces of the layered structure of the trial and true models are characterized by red and blue color. The multi-event stacking technique focuses these interfaces more and reduces ghost patterns, as compared with the image by the single event imaging. The offset due to the fault looks clearer in multi-event stacking. Comparison between the results of multi-event and singleevent analyses indicates that the patterns of images of a subsurface fault derived by both analyses show the same tendency for each trial model.

In the final step of flows in Fig. 1, we apply a simple image analysis by taking horizontal derivative to the image in Figs. 6 and 7 to pinpoint the offset due to the fault as shown in Fig. 8. The differentiation is made by the central difference. A strong contrast pair of red and blue spots corresponds to the fault, because the signal of the fault is characterized by positive vertical bands in the rigidity adjustment vector (see Murakoshi et al., 1996 for detail). We can find that two pairs of the red and blue spots are located around the upper and lower ends of the fault offset in Fig. 8. We can estimate the depth extent of the fault from these pairs.

We show an example of image derived by the multievent stacking for events with different source duration (i.e., different event size). Figure 9 shows the image obtained by the multi-event stacking of the data containing noise $(\mathrm{N} / \mathrm{S}=0.2)$ of the events with the incident angle $\pm 15^{\circ}$ and $\tau=0.5 \mathrm{~s}$ and $0.8 \mathrm{~s}$ using trial model 3. In Fig. 9, the interfaces of the trial and true structures are characterized by red and blue horizontal bands. From Fig. 9 we can see that the multi-event stacking technique is effective in detecting the location and size of a subsurface fault, even when signals with different pulse widths are stacked.

\subsection{Multi-master stacking}

We show the results for the multi-master stacking. In the multi-master stacking, the residual data $\delta v\left(\boldsymbol{x}_{r}, t\right)$ at the receiver locations in Eq. (2) depends on the selection of master receiver in the following way:

$$
\delta v\left(\boldsymbol{x}_{r}, t\right)^{(m)}=v_{\mathrm{ob}}^{\prime}\left(\boldsymbol{x}_{r}, t-t_{m}\right)-v_{\mathrm{th}}^{\prime}\left(\boldsymbol{x}_{r}, t\right),
$$

where $t_{m}$ is the time delay to synchronize the observed and the theoretical data, which maximize the correlation 


\section{$<\operatorname{model} 3, \mathrm{~N} / \mathrm{S}=0.0, \tau=0.5 \mathrm{~s}>$}
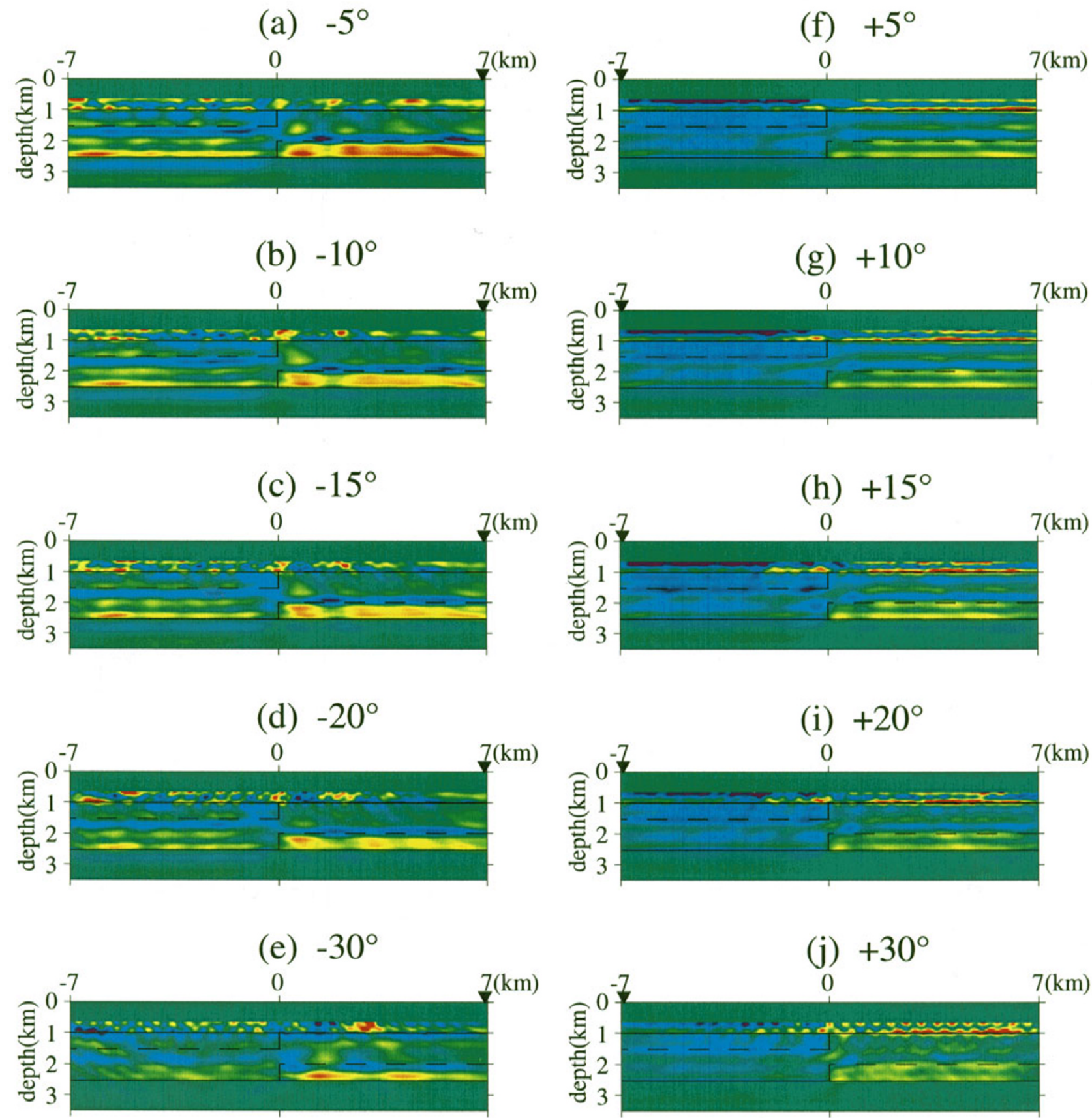

Fig. 5. The results of the single-event with the noise-free data of the event for the incident angle $\pm 5^{\circ}, \pm 10^{\circ}, \pm 15^{\circ}, \pm 20^{\circ}$ or $\pm 30^{\circ}$ and $\tau=0.5 \mathrm{~s} \mathrm{using}$ the trial model 3. From top to bottom, the incident angle $5^{\circ}, 10^{\circ}, 15^{\circ}, 20^{\circ}, 30^{\circ}$, and left and right are negative and positive incident angle. The red and blue colors indicate positive and negative adjustment, respectively. Since the target of our imaging is the fault, only the image in the area $-7 \leq x \leq 7(\mathrm{~km}), 0.6 \leq z \leq 3.5(\mathrm{~km})$ is shown. (The area $0 \leq z \leq 0.6(\mathrm{~km})$ is also painted with green for easy comparison with Fig. 2.) The solid and dashed lines are the same as in Fig. 4(c). "Master receiver" (solid triangle) has been here chosen as the receiver whose time of the initial phase is fastest.

between waveforms of the master receiver in observed and theoretical data. $v_{\text {ob }}^{\prime}$ and $v_{\text {th }}^{\prime}$ are the preprocessed waveforms of $v_{\mathrm{ob}}$ and $v_{\mathrm{th}}$, respectively, which are calculated using the corresponding waveforms of the master receiver (refer to Murakoshi et al., 1996). The steepest descent direction for the rigidity distribution in the multi-master stacking, if $N_{e}=1$ in Eq. (1), can then be obtained as

$$
\tilde{\delta \mu}(\boldsymbol{x})=-\sum_{m=1}^{N_{m}} \int_{0}^{T} d t \nabla v_{\mathrm{th}}(\boldsymbol{x}, t) \cdot \nabla \psi(\boldsymbol{x}, t)^{(m)}
$$

where $N_{m}$ is the number of master receivers and $\psi(\boldsymbol{x}, t)^{(m)}$ is obtained by solving Eq. (2) for each master receiver. 
$<\mathrm{N} / \mathrm{S}=0.0, \tau=0.5$, multi-event stacking $>$

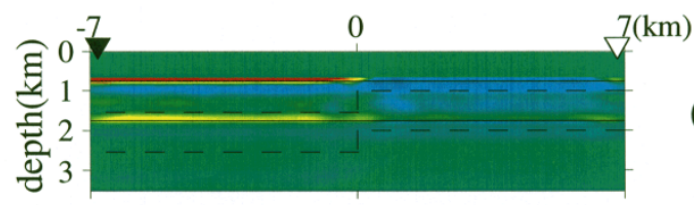

(a)

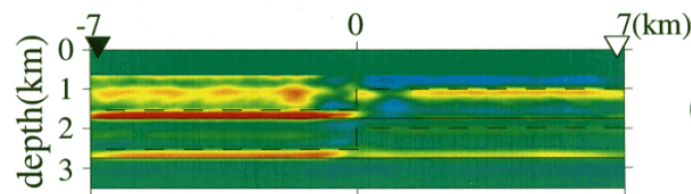

(b)

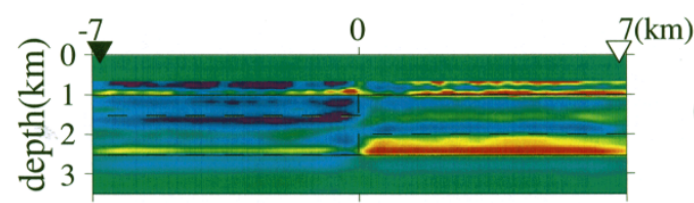

(c)
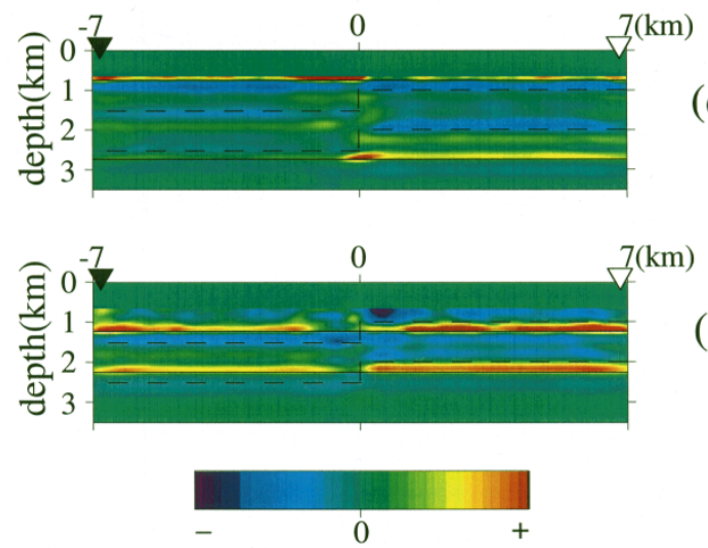

Fig. 6. The results of the multi-event stacking obtained from the noisefree data of the events for $\tau=0.5 \mathrm{~s}$ and incident angle $\pm 5^{\circ}, \pm 10^{\circ}, \pm 15^{\circ}$, $\pm 20^{\circ}$ and $\pm 30^{\circ}$ using the trial model 1 to 5 are shown in (a) to (e). "Master receiver" has been here chosen as the receiver whose time of the initial phase is fastest, which is denoted by the solid triangle (the incident angle $+5^{\circ},+10^{\circ},+15^{\circ},+20^{\circ}$ or $+30^{\circ}$ ) and the open triangle (the incident angle $-5^{\circ},-10^{\circ},-15^{\circ},-20^{\circ}$ or $-30^{\circ}$ ).
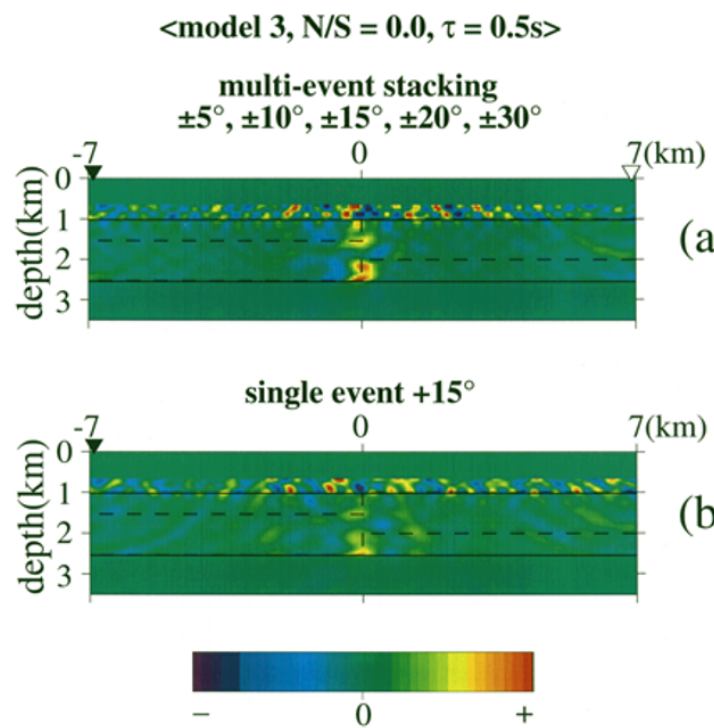

Fig. 8. Examples of the horizontal derivative of the steepest descent direction shown in Figs. 6(c) and 7(c). The red and blue spots are the signal from the fault.
$<\mathrm{N} / \mathrm{S}=0.0, \tau=0.5,+15^{\circ}$, single event $>$
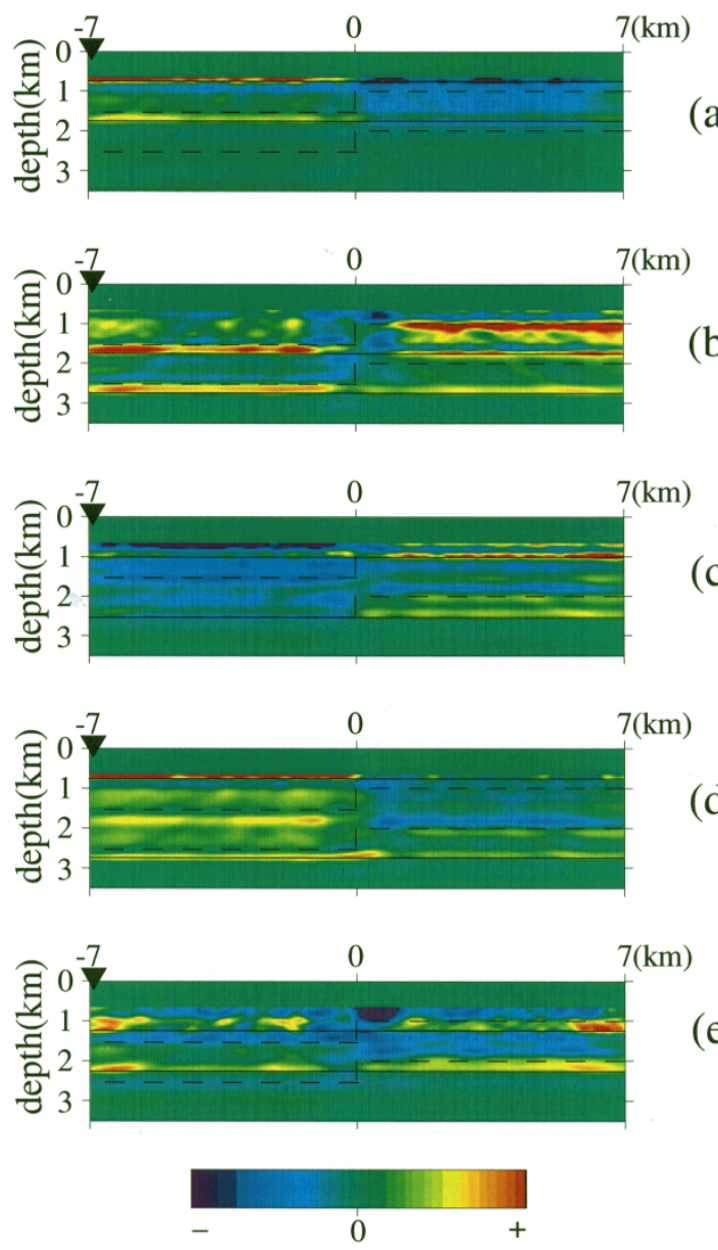

Fig. 7. The results of the single-event technique obtained from the data of the event for the incident angle $+15^{\circ}$ using the trial model 1 to 5 are shown in (a) to (e). The signal-to-noise ratio, the pulse duration and master receiver in (a) to (e) are the same as in Figs. 6(a) to (e), respectively. Symbol conventions are the same as in Fig. 5.

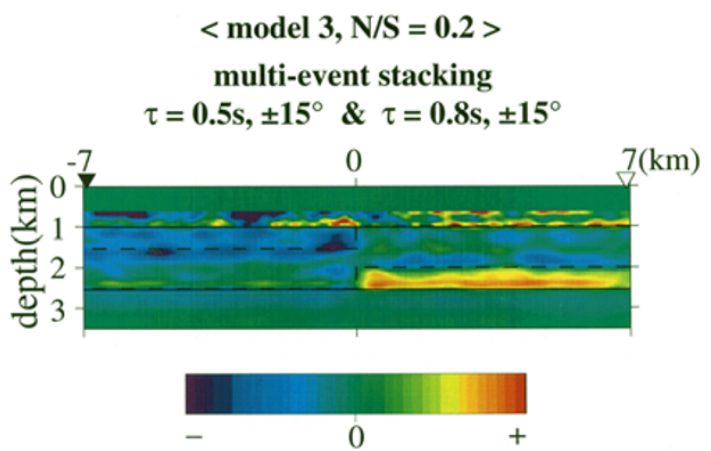

Fig. 9. The result of the multi-event stacking from the data containing noise $(\mathrm{N} / \mathrm{S}=0.2)$ of the events for the incident angle $\pm 15^{\circ}$ and $\tau=0.5$ and $0.8 \mathrm{~s}$ using the trial model 3. "Master receiver" has been here chosen as the receiver whose time of the initial phase is fastest, which is denoted by the solid triangle (the incident angle $+15^{\circ}$ ) and the open triangle (the incident angle $-15^{\circ}$ ). 

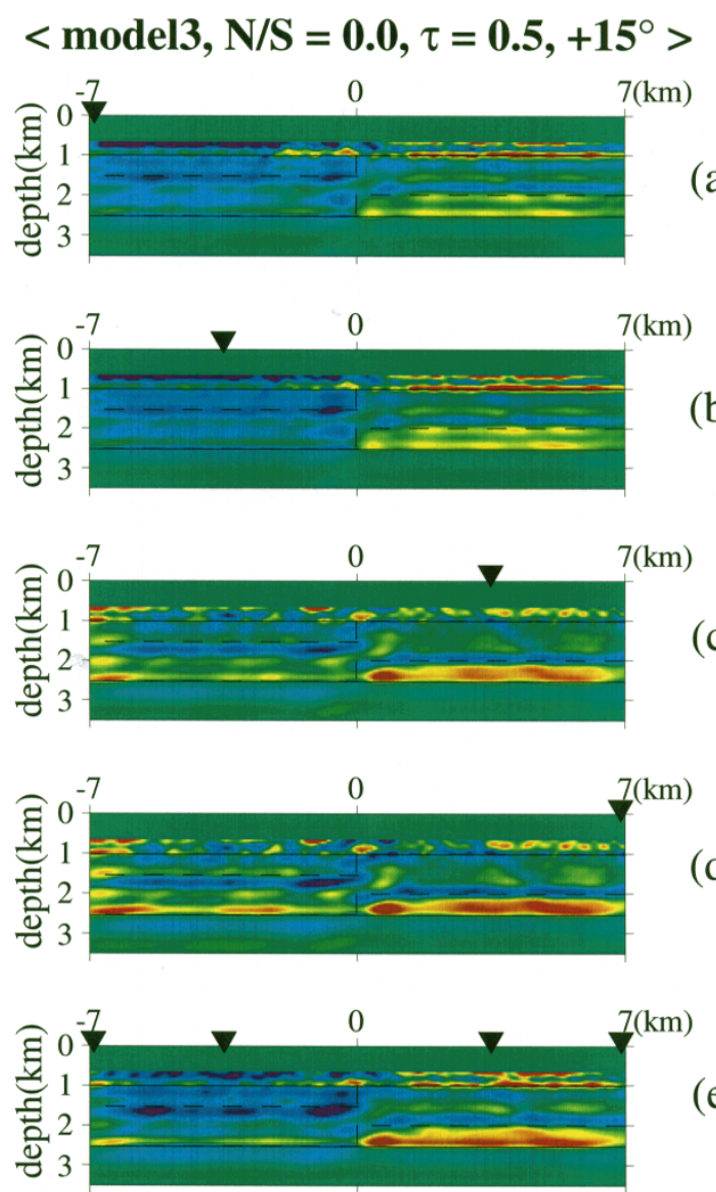

(e)

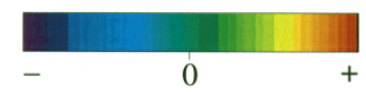

Fig. 10. The results of the single-event from the noise-free data for the incident angle $+15^{\circ}$ and $\tau=0.5 \mathrm{~s}$ using the trial model 3 and (a) $x_{\text {mas }}$ $=-7.0 \mathrm{~km}$, (b) $x_{\text {mas }}=-3.5 \mathrm{~km}$, (c) $x_{\text {mas }}=3.5 \mathrm{~km}$ or (d) $x_{\text {mas }}=7.0 \mathrm{~km}$. (e) The result of the multi-master stacking which is obtained by stacking the images (a)-(d). The "master receiver" is denoted by the solid triangle.

We now illustrate the process of the multi-master stacking in Fig. 10. Figures 10(a) to (d) are the images obtained from the noise-free data of a single event with the incident angle of $+15^{\circ}$ and $\tau=0.5 \mathrm{~s}$ using trial model 3 for the master receivers $x_{\text {mas }}=-7.0,-3.5,3.5$ or $7.0 \mathrm{~km}$, respectively, whereas Fig. 10(e) is the image obtained by the multi-master stacking which has stacked the images for all master receivers (i.e., Figs. 10(a) to (d)). Figure 10 reveals that the images obtained from the single-master data (Figs. 10(a) to (d)) have many ghost patterns, and the multi-master stacking reduces the ghost patterns (Fig. 10(e)), suggesting the multi-master stacking technique improves detectability of subsurface faults.

We consider the effect of data noise on the multi-master image. Figure 11 shows the images obtained by the multimaster stacking from the noise-free data (Fig. 11(a)), the data with noise of $\mathrm{N} / \mathrm{S}=0.1$ (Fig. $11(\mathrm{~b})$ ), $\mathrm{N} / \mathrm{S}=0.2$ (Fig. 11(c)) and N/S = 0.5 (Fig. 11(d)). The values of the incident angle and pulse duration and the trial model are the same as those in Fig. 10(e). The images of the layer

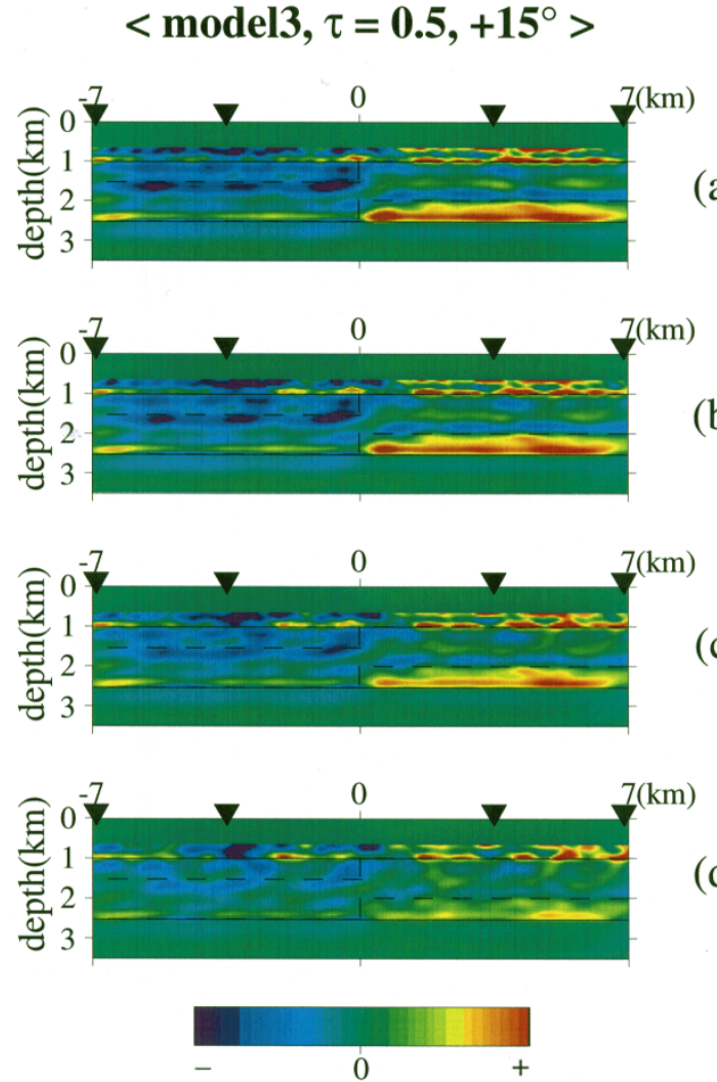

Fig. 11. The result of the multi-master stacking of (a) the noise-free data and the data containing noise, (b) N/S $=0.1$, (c) N/S $=0.2$ and (d) $\mathrm{N} / \mathrm{S}=0.5$. The value of the incident angle and the pulse duration and trial model are the same as in Fig. 10(e). The master receivers are denoted by the solid triangle.

$<\operatorname{model} 3, \mathrm{~N} / \mathrm{S}=0.0, \tau=0.5 \mathrm{~s},+30^{\circ}>$
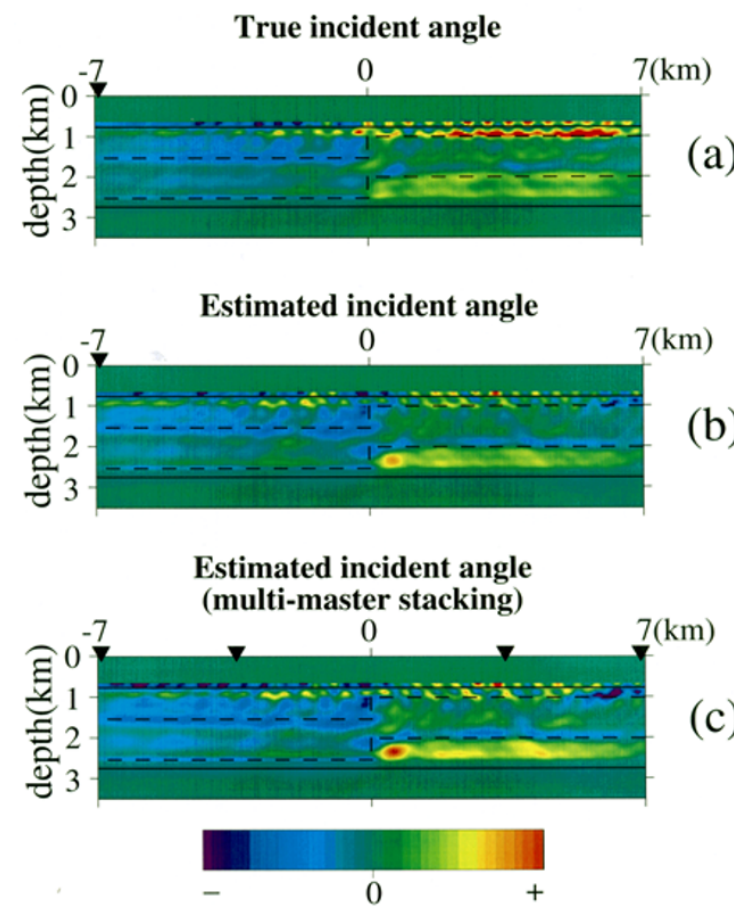

Fig. 12. The influence of the estimation error in the incident angle upon the resulting images. (a) The image obtained by a single master using the true $\left(+30^{\circ}\right)$ incident angle. Results of (b) single-master and (c) multi-master stacking using estimated $\left(+26.748^{\circ}\right)$ incident angle. Positions of the master receivers are denoted by the solid triangles. 
interfaces for $\mathrm{N} / \mathrm{S}=0.1$ and 0.2 are similar to that obtained by the multi-mater stacking for the noise-free data. However we cannot detect the location and size of a subsurface fault for $\mathrm{N} / \mathrm{S}=0.5$, since the reverberations due to the layer interfaces are completely masked over by noise as shown in Fig. 3(d).

\section{Discussion and Conclusion}

In real data processing, we should estimate the incident angle and the source wavefield as accurately as possible. Takenaka et al. (1996) used the same incident angle in the forward calculation as used in calculating the "observed" records. We have estimated the incident angle at the bottom of the laterally homogeneous trial model by the semblance analysis from the linear array data, which is now considered as the data of the master receiver and 4 receivers around the master receiver (Murakoshi et al., 1996). Table 1 shows examples of estimated incident angle in case of the data for $\mathrm{N} / \mathrm{S}=0.0, \tau=0.5 \mathrm{~s}$ and incident angle of $+5^{\circ},+10^{\circ},+15^{\circ},+20^{\circ}$ or $+30^{\circ}$ using trial model 5 . The error in the estimated incident angles are less than $10 \%$ for all the incident angles. The errors for different combination of N/S and $\tau$ are comparable to those in Table 1. To see the influence of the error in the incident angle estimation upon the resultant images, we show the single-event images of the noise-free data of an event (incident angle of $+30^{\circ}$ and $\tau=0.5 \mathrm{~s}$ ) for trial model 5 using the true and estimated incident angles in Figs. 12(a) and (b), respectively. The fault is reasonably well detected using the estimated incident angle, while the interfaces imaged in Fig. 12(b) are broadened as compared with those for the true incident angle (Fig. 12(a)). The effect of error in the incident angle estimation is smaller for steeply incident waves, since the difference between the true and estimated incident angles is small. Figure 12(c) is the image obtained by the multi-master stacking which has stacked the images for the master receiver $x_{\mathrm{mas}}=-7.0$ (Fig. 12(b)), $-3.5,3.5$ and $7.0 \mathrm{~km}$ using the estimated incident angles. The multi-master stacking technique focuses these interfaces more and reduces ghost patterns, as compared with the images by the single event imaging (Figs. 12(a) and (b)). From Fig. 12 we can see that our stacking technique is effective in detecting the location and size of

Table 1. The estimated incident angles in case of the noise-free data for the event for the incident angle $+5^{\circ},+10^{\circ},+15^{\circ},+20^{\circ}$ or $+30^{\circ}$ and $\tau=0.5 \mathrm{~s}$ using the trial model 5 .

\begin{tabular}{cc}
\hline True incident angle & Estimated incident angle \\
\hline $5.0^{\circ}$ & $5.483^{\circ}$ \\
$10.0^{\circ}$ & $10.869^{\circ}$ \\
$15.0^{\circ}$ & $15.097^{\circ}$ \\
$20.0^{\circ}$ & $18.572^{\circ}$ \\
$30.0^{\circ}$ & $26.748^{\circ}$ \\
\hline
\end{tabular}

a subsurface fault, even when the data contain the error in the estimated incidence angle.

The objective of this study is to improve the imaging method for sensing the subsurface fault, and to investigate the applicability of the proposed techniques: the multievent stacking and the multi-master stacking. Both the techniques work reasonably well to improve the detectability of subsurface faults. Although we have used only four master receivers for the multi-master stacking, we could obtain much better image of a subsurface fault than that by a single-master receiver. There is no problem in using both techniques simultaneously, for which we expect even more improvement in the detectability than demonstrated in the present paper.

Acknowledgments. We would like to thank Dr. F. Haslinger and Dr. T. Yokokura for careful reviews and constructive comments.

\section{Appendix. Design of Noisy Observed Records}

Here, we describe the design of noisy observed records step by step.

[1] We first produce spectral data with constant power and random phase for each record.

[2] We multiply the power spectra produced in step [1] by the power spectrum of the signal to obtain "observed noisy data" using inverse FFT. The power spectral shape of noise is then identical with that of signal, which is the worst condition for detecting reverberation due to a layered structure.

Figures A1(a), (b), and (c) show an example of noise at $x_{r}=-7 \mathrm{~km}$, the noise-free signal, and the noisy record made by summing (a) and (b), respectively. The N/S value of this noisy record is 0.5 . In the imaging procedure we use noisy records as shown in Fig. A1(d) by tapering out the initial phase as mentioned in Section 3.

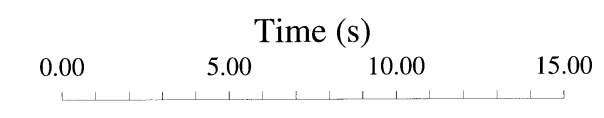

(a)

(b)

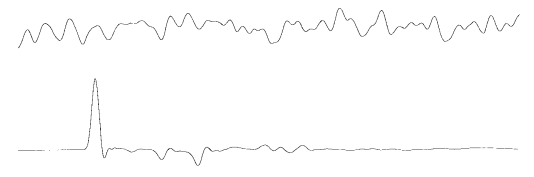

(c)

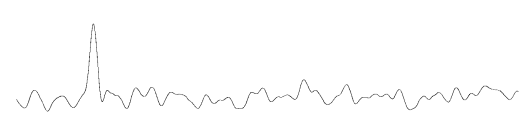

(d)

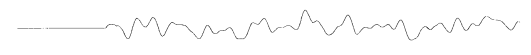

Fig. A1. Example of (a) a noise, (b) a noise-free signal, (c) noisy record, and (d) a preprocessed waveform, where the initial $S H$ phase has been tapered out. 


\section{References}

Boore, D. M., Finite difference methods for seismic wave propagation in heterogeneous materials, in Methods in Computational Physics, edited by B. A. Bolt, pp. 1-37, Academic Press, New York, 1972.

Gauthier, O., J. Virieux, and A. Trantola, Two-dimensional nonlinear inversion of seismic waveforms: numerical results, Geophys., 51, 1387-1403, 1986.

Kong, S. M., R. A. Phinney, and K. Roy-Chowdhury, A nonlinear signal detector for enhancement of noisy seismic record sections, Geophys., 50, 539-550, 1985.

Murakoshi, T., H. Takenaka, D. Suetsugu, and T. Saita, Imaging of subsurface faults using teleseismic waveform-Numerical experiments-, Zisin, 49, 389-401, 1996 (in Japanese).

Takenaka, H., T. Murakoshi, D. Suetsugu, and T. Furumura, An imaging technique for subsurface faults using teleseismic-wave records- $\mathrm{SH}$ case-, J. Phys. Earth, 44, 529-541, 1996.

Tarantola, A., Inversion of seismic reflection data in the acoustic approximation, Geophys., 49, 1259-1266, 1984.

T. Murakoshi (e-mail: murakoshi@geo.kyushu-u.ac.jp), H. Takenaka (e-mail: takenaka@geo.kyushu-u.ac.jp), and D. Suetsugu (e-mail: dai@kenken.go.jp) 